\title{
Alpha-1- Antitrypsin Deficiency Liver Disease: Science and Therapeutic Potential 50 Years Later
}

\author{
(A Report from an International Research Conference on Alpha-1-AT) \\ Pradipta Chakraborty ${ }^{1}$ and Jeffrey Teckman ${ }^{1,2 *}$ \\ 1Department of Biochemistry and Molecular Biology, Saint Louis University School of Medicine, USA \\ 2Department of Pediatrics, Saint Louis University and Cardinal Glennon Children's Medical Center, USA
}

\begin{abstract}
Received: July 18, 2014; Accepted: September 03, 2014; Published: October 08, 2014
*Corresponding author: Jeffrey Teckman, St. Louis University School of Medicine, Cardinal Glennon Children's Medical Center, 1465 South Grand, Blvd, St. Louis, MO 63104, USA, Tel: 314-577-5647; E-mail: teckmanj@slu.edu
\end{abstract}

\begin{abstract}
Alpha-1-Antitrypsin (A1AT) deficiency is a genetic disease first described in 1963 by Laurell and Eriksson. It was recognized as a cause of emphysema in adults, and later was also identified as a cause of chronic liver disease, cirrhosis and hepatocellular carcinoma in children and adults. Subsequent reports have documented the highly variable spectrum of clinical severity in this disease, which leaves the lung vulnerable to damage due to a loss-of-function mechanism from low levels of circulating A1AT. However, liver injury is due to accumulation of the A1AT mutant Z protein in the liver via a toxic, gain-of-function mechanism. Recent scientific insights have not only explained many fundamental aspects of liver injury in this disease, but have also allowed new methods of therapy to be proposed. Several new clinical trials are the result. These studies have included descriptions of how the accumulation of the mutant $\mathrm{Z}$ protein within hepatocytes triggers apoptotic cell death in the few hepatocytes with the greatest mutant protein burden. Furthermore, protein degradation pathways within hepatocytes which act to degrade the accumulated mutant $\mathrm{Z}$ protein as protective mechanisms are attractive targets for the development of new therapies. In observance of the 50 years since the disease was first discovered, an International Conference on Alpha-1-antitrypsin liver disease was held on April 11-12, 2013 in Barcelona, Spain. Sessions included examination of new scientific insights into disease mechanisms, new liver therapeutics and the challenges of human trials in liver disease. The new observations presented not only fill gaps in the understanding and treatment of this metabolic disease, but also suggest new approaches to many general aspects of hepatocellular protein processing and liver injury.

Keywords: Liver; Autophagy; ERAD; Apoptosis; Anti-sense oligonucleotide
\end{abstract}

\section{Introduction}

Alpha-1-Antitrypsin (A1AT) deficiency is a common genetic disease found predominantly in North American and European populations. In the classical form, patients are homozygous for the Z mutant of the A1AT gene, so called ZZ or PIZZ. A1AT deficiency can cause liver disease in adults and children, and lung disease in adults. The disease has highly variable clinical manifestations and as a result, is widely misunderstood. In recent decades, groups of patients and physicians from around the world have met from time to time to discuss patient care and scientific advances. In 2013 such an international meeting was convened in commemoration of the 50 years since the disease's first description. Here we present a review of the current state of knowledge supplemented with new data from the scientific presentations made during this historic meeting.

\section{Historical descriptions of genetics and clinical course}

A1AT deficiency has a complex pathophysiology, is highly variable in clinical course, and is under diagnosed. The association with chronic lung disease was first described by Eriksson and Laurell in 1963, and later, Sharp and colleagues recognized A1AT deficiency as a cause of liver disease. In 2013 an international meeting was held to review the intervening 50 years of basic and clinical science, with a focus on liver disease $[1,2]$. Leaders in basic science investigation and in clinical medicine presented both retrospective commentary and new data relating to A1AT liver disease. A1AT is the archetype of the Serine Protease Inhibitor (SERPIN) family and is encoded by the gene SERPINA1. A1AT protein is produced in the liver and secreted in the serum in large quantities. The function of $\mathrm{A} 1 \mathrm{AT}$ is to inhibit neutrophil proteases released non-specifically during periods of inflammation [1-4]. Over 100 variant alleles of the A1AT gene have been described but the overwhelming majority of patients with liver disease are homozygous for the $\mathrm{Z}$ mutant allele. Homozygosity for this autosomal co-dominant Z mutant of A1AT, referred to as ZZ or "PIZZ" in World Health Organization nomenclature, is the classical form of A1AT deficiency. The mutant $Z$ protein accumulates within hepatocytes rather than being efficiently secreted (see below). This results in a lower, "deficient" level of protease inhibitor activity in serum. Within the hepatocyte, the $\mathrm{Z}$ mutant protein accumulates in the Endoplasmic Reticulum (ER), and may attain an altered conformation in which many A1AT mutant Z molecules aggregate to form large polymers. ZZ homozygous adults have a markedly increased risk of developing emphysema by a loss-of-function mechanism in which insufficient circulating A1AT is available in the lung to inhibit non-specific connective tissue breakdown, which can occur during granulocyte phagocytosis. A subgroup of ZZ homozygous children and adults may also develop liver disease and Hepatocellular Carcinoma [HCC] via a toxic, gain-of- 
function mechanism in which the intracellular accumulation in the liver of A1AT mutant $\mathrm{Z}$ protein triggers cell death and chronic liver injury [5,6](Figure 1 ).

Homozygous ZZ individuals occur in 1 in 2,000-3,500 births in North America and Europe, making it one of the most common single gene diseases in these populations. Manifestations of liver disease can appear in ZZ individuals at any age. Some neonates present with the "neonatal hepatitis syndrome", characterized by biochemical hepatitis and cholestatic jaundice. The majority of these infants recovers spontaneously and remains healthy throughout childhood, but some progress to cirrhosis, liver failure and death or liver transplant. Older children may develop hepatomegaly, chronic hepatitis or cirrhosis, even if they have not previously had clinically detected liver disease as infants. The risk of life-threatening liver disease in childhood may be as low as $5 \%$, although the incidence of any sign or symptom of liver disease, such failure to thrive or elevated transaminases, may be as high as $50 \%$. Liver disease is thought to increase in incidence with advancing age in adulthood. Some autopsy studies suggest the life-long risk of cirrhosis may be as great as $40-50 \%$.

The seminal study of the clinical course of A1AT deficiency was the birth cohort study undertake in Sweden in the 1970s by Sveger and colleagues [7]. More than 200,000 newborns were screened and $127 \mathrm{ZZ}$ and $54 \mathrm{SZ}$ infants were identified, as well as other groups of various genotypes. Much of the understanding of the variable nature of $\mathrm{ZZ}$ children, and the benign course of the majority of these children, comes from this study. Eeva Piitulainen [8] presented an update on the Swedish birth cohort for this conference, whose participants are now over 40 years of age. The findings of $17 \%$ of $\mathrm{ZZ}$ infants with neonatal liver disease in the cohort and 4-5\% mortality of the cohort (pre-liver transplant era) were reviewed. The cohort continues to be followed through medical records on a regular schedule, but direct contact between subjects and investigators is inconsistent. A small percentage has been lost to follow up, and unrecorded deaths or liver transplants cannot be ruled out. No evidence of liver disease is reported in surviving subjects available for follow up since childhood, based on physical exams and blood tests recorded in reviewed charts, and no additional deaths in subjects have been recorded. However, standardized exams, imaging studies and liver biopsies have not been performed. Overall, the rate of elevated ALT in the ZZ subjects is similar to that of the Swedish general population (Figure 2). This is surprising as anecdotal experience in the US and in other centers in Europe is that ALT elevation is very common in ZZ patients, even when there is minimal liver injury. Three of 54 SZ patients have died, although the rest are healthy, except for mild ALT and AST elevations. The three SZ deaths had various liver abnormalities recorded, including steatosis and one with cirrhosis, but drug and alcohol abuse appeared to have played a role. This outstanding cohort study is remarkable in scope and length, but also in the very low rate of liver disease observed. It is unclear if the low rate of disease is applicable to all populations of ZZ patients, or if the environmental or genetic modifiers present in the Swedish population are less injurious that those found in more heterogeneous populations such as the United Kingdom or North America.

\section{Discovery of cellular mechanisms of ZZ liver disease}

In the 50 years since this disease was first described there has been an evolution in understanding of how accumulation of the mutant $\mathrm{Z}$ protein in hepatocytes triggers liver injury. A few seminal observations have driven the field. First, was the original recognition by Sharp et al. [9] that ZZ patients develop liver disease. Then, two decades later, studies of patient-derived fibroblast cell lines by Perlmutter and colleagues showed reduced intracellular clearance of mutant $\mathrm{Z}$ protein correlated to life-threatening liver disease, which gave the strongest support up to that time that accumulation of the mutant $\mathrm{Z}$ protein in the liver was the key trigger of liver injury [10]. Further studies from several laboratories, including Sifers, provided critical information on the mechanisms of ER-Associated Degradation (ERAD), and how this important housekeeping function in many cell types is uniquely related to the pathophysiology of A1AT

\section{Human ZZ Liver}

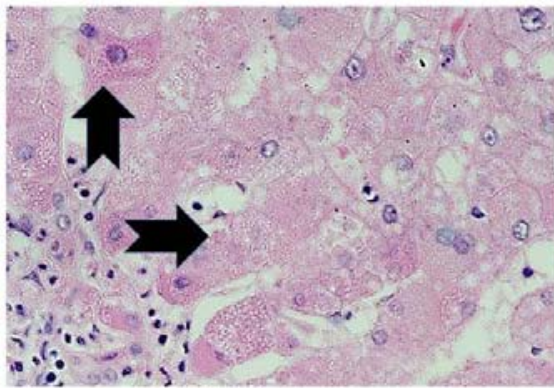

H\&E

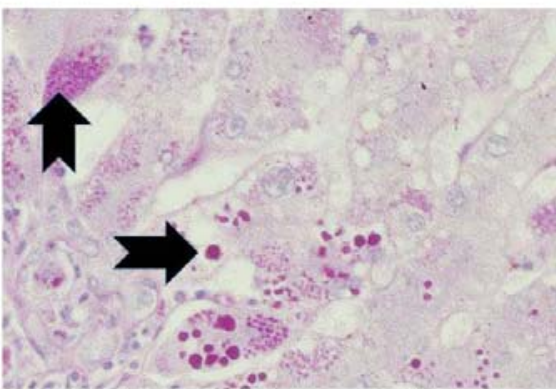

PAS+Digestion

Figure 1: Photomicrograph of human ZZ liver serial sections stained with H\&E (left panel) and PAS with digestion (right panel). Arrows show various sizes of "globular" inclusions of A1AT mutant Z polymerized protein. 


\title{
Elevated plasma ALT in adulthood
}

\begin{tabular}{|c|c|c|c|}
\hline Age & PiZZ & PiSZ & $\begin{array}{c}\text { Controls } \\
(\text { PiMM) }\end{array}$ \\
\hline 22 years & $7 \%$ & $17 \%$ & - \\
\hline 26 years & $7 \%$ & $13 \%$ & - \\
\hline 30 years & $5 \%$ & $10 \%$ & $6 \%$ \\
\hline 34 years & $7 \%$ & $11 \%$ & $5 \%$ \\
\hline
\end{tabular}

${ }^{*}$ Random sample from the population registry

Figure 2: Percent of ZZ and SZ subjects with ALT elevations at follow up intervals as shown, in the Swedish A1AT birth cohort compared to normal MM controls in Sweden (figure provided by Dr. Eeva Piitulainen). Comparison of all values $p>0.05$.

\author{
Hepatocellular Liver Injury Cascade \\ alATZ protein synthesis \\ a1ATZ protein ER retention $\Rightarrow$ ERAD proteolysis \\ $1 ? ?$ ? \\ alATZ protein polymerization $\Rightarrow$ Autophagic degradation \\ 1 \\ Heterogeneous hepatocyte alATZ polymer accumulation \\ Caspase activation, mitochondrial \\ changes, redox injury in \\ hepatocytes with large polymers \\ Apoptotic and other death pathways \\ Hepatocytes with low polymer \\ proliferate to maintain liver mass \\ I \\ Chronic regenerative stimulus

$\begin{gathered}\text { Environmental-Genetic } \\ \text { modifiers }\end{gathered}$
Cell death $\rightarrow \begin{gathered}\text { Chronic hepatocellular death and } \\ \text { regeneration leads to fibrosis and HCC } \\ \text { Liver Death }\end{gathered}$

Figure 3: Hypothetical liver injury cascade in PIZZ A1AT deficiency. The A1AT mutant Z protein is appropriately synthesized, but then retained in the ER of hepatocytes rather than being secreted due to abnormal folding. Quality control processes within the cells direct most of these abnormal, mutant Z protein molecules into intracellular proteolysis pathways related to the proteasome (ERAD). However, some of the mutant $\mathrm{Z}$ protein molecules escape proteolysis and attain a unique, polymerized conformation forming inclusions in the ER. Autophagic degradation is upregulated to cope with the mutant $\mathrm{Z}$ polymer accumulation. For reasons that are not clear, a small population of hepatocytes develops especially large accumulations of polymerized mutant $\mathrm{Z}$ protein and undergo cell death involving apoptosis and other mechanisms. The hepatocytes with a smaller burden of mutant $\mathrm{Z}$ protein proliferate, possibly with the input of a liver stem cell population, to maintain the functional liver mass. This chronic process of injury, cell death, and compensatory proliferation is known to lead to end organ processes of fibrosis, cirrhosis, and HCC. Given the variable nature of clinical liver injury between individuals with the same genotype, and the usually slow disease progression, there are likely to be important environmental and genetic disease modifiers affecting the rate and magnitude of these processes.

deficiency $[11,12]$. Several mouse models of ZZ liver disease have been created, but the PiZ mouse developed 25 years ago by Woo and colleagues has been an invaluable resource for the study of injury pathways and to investigate therapeutic strategies. At the same time, the polymerized conformation of the mutant $\mathrm{Z}$ protein was discovered by Lomas and Carrell [13], which focused the field on the key concept of protein conformation. More recently was the discovery by Teckman and Perlmutter [14] that autophagy was an important route of intracellular degradation for the mutant $\mathrm{Z}$ protein, which when combined with these 
other concepts has led to multiple new therapeutic approaches. Finally, Teckman and Perlmutter, described how hepatocellular apoptosis and compensatory proliferation in the liver, related to mutant $\mathrm{Z}$ protein accumulation was linked to cirrhosis and HCC $[4,5]$. Conference attendees explored in detail new data relating to these key concepts.

\section{The intracellular molecular injury cascade}

During biosynthesis the A1AT mutant Z gene is appropriately transcribed, translated, and then the nascent mutant $\mathrm{Z}$ polypeptide chain is translocated into the ER lumen. However, in the ER the mutant $\mathrm{Z}$ protein molecule folds slowly and inefficiently into its final, secretion-competent conformation $[10,13,15-18]$. A system of proteins within the ER, termed the "quality control" apparatus, recognizes these mutant Z molecules as abnormal and directs them to a series of proteolytic systems rather than allowing progression down the secretory pathway $[10,11,14,17,19,20]$. However, this process of quality control holds the mutant $\mathrm{Z}$ molecules in the ER lumen for a longer time than during secretion of the wild type $\mathrm{M}$ protein. For reasons that are not clear, but which might be related to this "lag" in degradation, some of the mutant $\mathrm{Z}$ molecules escape proteolysis and may attain a variety of abnormal conformations including a unique state in which multiple molecules aggregate to form large, stereotypic and repeating quaternary structures referred to as "polymers" (discussed further below) [13,16,21]. This polymer conformation is highly thermodynamically stable and links large groups of mutant $\mathrm{Z}$ molecules together with non-covalent bonds. These polymers have a long biological half-life within cells. Accumulations within hepatocytes of the polymerized mutant $\mathrm{Z}$ protein may be large enough to be seen under light microscopy and represents the hepatocellular "globules" observed in the ZZ liver (Figure 1). The result of these processes is that only approximately $15 \%$ of A1AT mutant Z protein molecules are secreted into the serum. The hepatocytes with the largest mutant $\mathrm{Z}$ polymer accumulations undergo apoptosis and other hepatocytes proliferate to replace them. This chronic process of hepatocellular death and regeneration eventually leads to organ injury, fibrosis and HCC (Figure 3).

\section{Treatment Options, New Science and Meeting Presentations}

At present, there is no specific treatment for liver disease associated with A1AT deficiency. A1AT lung disease is often treated with one of several serum protein replacement products, but since liver disease is not related to the serum deficiency, protein replacement has no role in treating liver disease. Liver treatment is based on supportive care for typical liver failure and portal hypertension. This includes nutritional support for underweight patients or those with fat soluble vitamin deficiency, support for liver synthetic dysfunction, treatment of cholestatic itching, if present, and management of variceal bleeding, hepatopulmonary syndrome or hepatorenal syndrome. Liver transplant is an option for patients with decompensated cirrhosis. A range of meeting presentations were made, which included the application of new discoveries to possible new therapies.

\section{Protein Polymerization}

Like other SERPINs, A1AT remains in a metastable state [22]. Metastability of protein means that the native fold of the WT is not the most thermodynamically favorable form that could be achieved by its primary amino acid sequence. The most conformationally stable fold can be achieved, rather, when the protein interacts with its substrate molecule. Due to this unique aspect of metastability, all SERPINs including A1AT have the tendency to become polymerogenic in the presence of a subtle change in the primary amino acid sequences. Steven Bottomley [23] presented new concepts of serpin misfolding and its role in serpinopathies. Studying the effect of the $\mathrm{Z}$ mutation on the structure and thermodynamic stability of A1AT may permit the design drugs to prevent the formation of such toxic aggregates. Bottomley and other research groups [21] have shown previously that both the A1AT WT M and mutant Z proteins have three step conformational stages, designated as Native $(\mathrm{N})$, Intermediate (I) and Unfolded (U) states. He presented evidence which suggests that misfolded A1AT achieves an Intermediate (I) conformation that is highly polymerogenic in nature. Extensive biophysical and biochemical studies have shown the structural basis of polymer formation in $\mathrm{Z}$ mutants. These data suggest that although the thermodynamic stability of the native state of WT and mutant Z A1AT are similar, that the kinetics of transforming $\mathrm{N}-\rightarrow$ I in AAT Z is 1.5 times faster than WT, while the second transition kinetics remains unaltered. The observation is that the $\mathrm{Z}$ mutation decreases the kinetic barrier of first transition state while not affecting the second is significant. As a consequence, more polymerogenic intermediates are formed in Z than WT at any given time. Bottomley has performed screening of Small Heat Shock Protein (SHSP) molecules that may be able to increase the activation barrier of the conversion of A1AT Z $(\mathrm{N})$ form to (I) form. These SHSPs will therefore, result in the formation of more monomeric misfolded A1AT Z instead of polymerogenic intermediate forms, and in the future might be developed as medicinal drugs.

\section{Intracellular proteolysis}

Once the mutant $\mathrm{Z}$ protein is retained in the $\mathrm{ER}$, the hepatocyte attempts to deal with this burden of unfolded protein via intracellular pathways for protein degradation. These include ubiquitin dependent and ubiquitin independent proteasomal pathways, and possibly other mechanisms [11,20,24,25]. These pathways are sometimes referred to as "ER Associated Degradation" (ERAD), and are thought to be critical mechanisms for liver cells to "protect" themselves from the accumulation of abnormally folded proteins. It is thought that these pathways are the primary route for degradation of A1AT mutant Z in the non-polymerized conformation. These proteolytic pathways successfully process the vast majority of A1AT mutant Z protein molecules retained within the ER. Although many of the mechanistic steps in the degradation process, and their specific 
sequence, are still under investigation, Richard Sifers presented data that two molecules present in the ER, calnexin and ER mannosidase I (ERmanI), are likely to be critical points of control $[26,27]$. Calnexin is a transmembrane ER chaperone which binds A1AT mutant Z, becomes targeted for degradation by linkage to ubiquitin, and then is degraded as this trimolecular complex (A1AT mutant Z-calnexin-ubiquitin) by the proteasome [17]. Studies by Teckman and Perlmutter $[10,28]$ in human fibroblast cell lines established from ZZ homozygous patients show that patients susceptible to liver disease have less efficient ER associated degradation of a1AT mutant Z protein than ZZ patients without liver disease. The reduced efficiency of degradation in the liver disease patients presumably leads to a greater steady state burden of mutant $\mathrm{Z}$ protein within liver cells and increased liver injury. Similarly, studies of the enzyme ERmanI by Sifers suggest that it also may have a critical role in directing A1AT mutant Z molecules to the proteasome for degradation. These data raise the possibility that allelic variations in calnexin, ERmanI, or in other proteins involved in the quality control or proteolytic systems might alter susceptibility to liver injury by changing the efficiency of degradation [19,29].

Richard Sifers [30] presented his recent observation of the Single Nucleotide Polymorphism (SNP) in ERmanI that makes ZZ individuals susceptible to early life liver cirrhosis [Figure 4]. He also presented new information regarding the cellular localization of the enzyme and its mechanism in targeting the A1AT Z proteins. Experimental evidences suggest that a SNP can lead to decreased expression of ERmanI under the condition of ER stress. This SNP designated as rs4567 that contains A instead of $G$, results in the suppression of ERmanI under the condition of ER stress. Such, homozygosity of rs 4567 A has been reported in ZZ infants who suffered from chronic liver injury. Current studies by Sifers revealed the mechanism of Golgi localized ERmanI mediated quality control. By using classical misfolded protein Null Hong-Kong (NHK) variant of A1AT, he has shown that ER man 1 localized in the Golgi interacts directly with the COP1 component of vesicle formation via its cytoplasmic tail. Disruption of this interaction results in decreased degradation and increased secretion of the misfolded proteins. This observation points out that there are limitations in the level of ER retention of misfolded protein and once the threshold is reached, the protein is no longer retained and is secreted.

Like all secretory glycoproteins, A1AT biogenesis is regulated by the Proteostasis Network (PN) prior to its successful secretion into the serum [31]. The network is constituted of a complex array of chaperones, folding enzymes, and degradation machineries. The correction of A1AT Z conformational abnormality by cellular $\mathrm{PN}$ is hindered and subsequently resulted in the retention of A1AT in the ER of the hepatocytes, as proposed and presented by William Balch [31]. He reported his research on understanding the function of the PN and controlling the activity of PN under misfolded secretory glycoprotein (e.g CFTR F508 and A1AT Z) retention in ER. He reviewed that the PN is controlled by several pathways, including the unfolded protein response, heat shock response, calcium sensing signaling pathways, autophagy, oxidative stress signaling, and acetylation proteostasis system [32]. Hence it is necessary to manipulate PN in a way so that the misfolded proteins are now capable of being properly folded and secreted by PN. To this end, he has studied extensively the effect of small molecules on PN in the context of misfolded protein diseases. He presented data on the effect of Suberoylamilide Hydroxamic Acid (SAHA), which is a potent HDAC inhibitor resulting in increased folding, maturation, and secretion of the misfolded A1AT Z. This is an observation with a high potential for therapeutic development. However, addition of SAHA also results in the increased translation of A1AT WT and Z mutants. Biochemical studies suggest that corrected folding due to SAHA treatment occurs via disruption of calnexin-A1AT Z interaction. Further development of SAHA may lead to a therapeutic intervention for liver disease.

\section{The role of autophagic protein degradation}

Autophagy is a highly conserved cellular pathway involved in the clearance of abnormal proteins, and the disposal of senescent organelles, among other functions. Autophagic degradation involves the formation of unique, double membrane bound cytoplasmic vacuoles, which arise from membranes associated with the endoplasmic reticulum. These vacuoles incorporate the targets of degradation and then mature and fuse with lysosomes, and other structures, to complete the destruction of the contents. Autophagy is an important route of the disposal of the toxic, A1AT mutant $\mathrm{Z}$ protein polymers within liver cells. Data suggests that the large polymers are insoluble and difficult for the cell to manage, and therefore are a poor substrate for ERAD. Autophagy however, is designed to handle bulk input, including whole organelles, which may explain the utility of autophagy for disposal of A1AT mutant Z protein polymers. Several studies in experimental systems show that liver injury can be reduced by enhancing autophagic degradation of mutant $\mathrm{Z}$ protein. This is similar to the role of ERAD in this disease, although ERAD is thought to play a larger role in degradation of monomeric A1AT mutant Z molecules. Published studies of rapamycin and carbamazepine in mouse models have shown in vivo proof of concept that drugs which enhance autophagy ameliorate liver damage in the model PiZ mouse [33-35]. Human studies of the possible use of carbamazepine are ongoing, but are only recommended in research settings. One study is recruiting exclusively ZZ patients with end stage cirrhosis and is using only $10 \%$ of the $\mathrm{mg} / \mathrm{kg}$ dose in humans as was found therapeutic in the mouse studies. Results are not yet available. Rapamycin has not been examined in humans for this indication, to date, due to concerns about toxicity. Nicola Brunetti-Pierri [35] discussed recent advancements made in his laboratory on how to enhance autophagy using gene expression techniques. His study focuses on the effect of Transcription Factor EB (TFEB). TFEB is known to be the master regulator of autophagy and lysosomal biogenesis. It has been shown that TFEB can increase the number of autophagolysosomes, induce expression of autophagy genes, and results in the clearance of the lysosomes in mice. Therefore, 


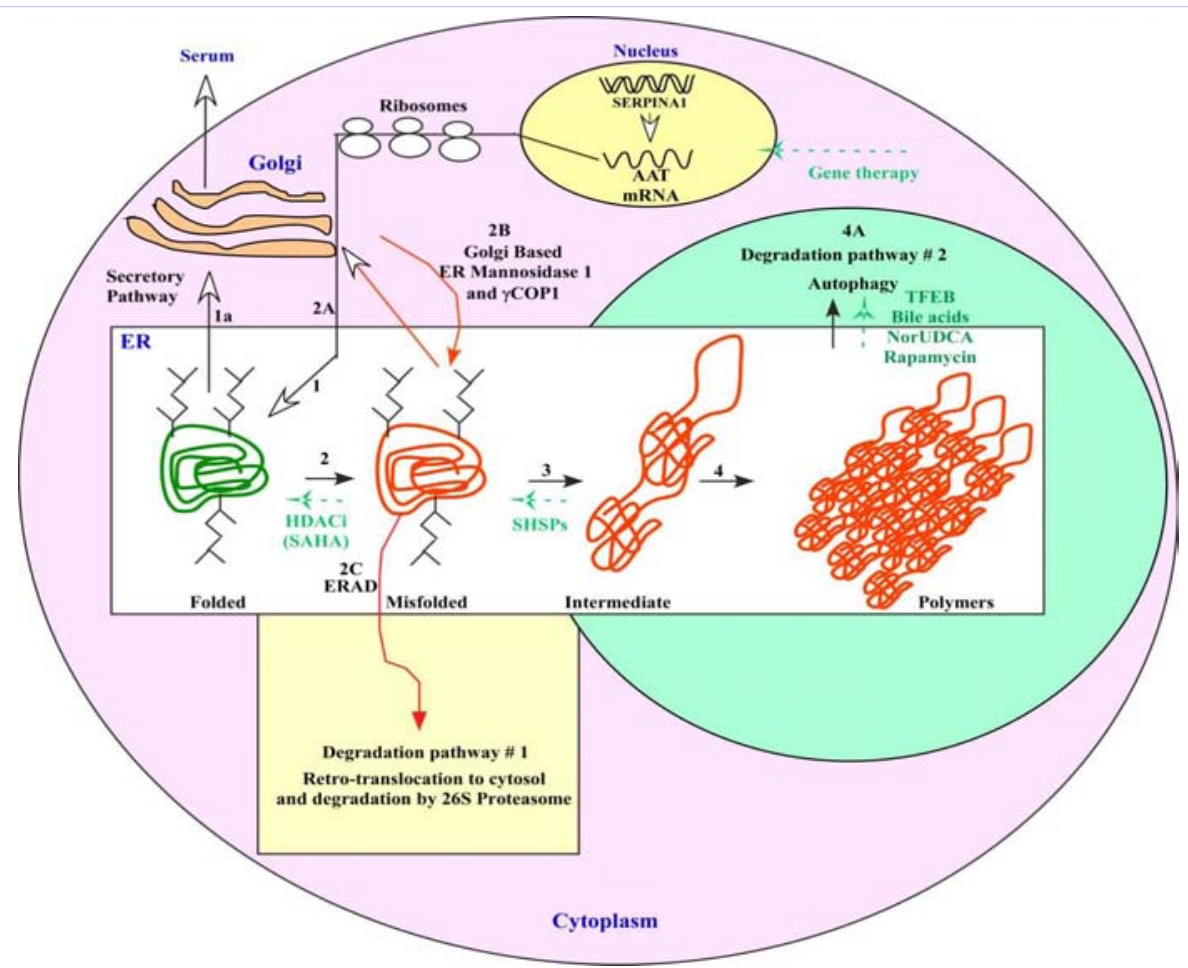

Figure 4: Stages of AAT-Z mediated liver injury and potential therapeutic strategies. 1. AAT mRNA is transcribed from SERPINA1gene in nucleus and translated to AAT polypeptides sequence. AAT nascent polypetide translocates to the ER, where by cellular proteastasis network folds properly to its native structure and goes through secretory pathway via golgi and secreted to serum. 2 . Point mutation E342K in SERPINA1 gene results in the production of mutated polypeptide that misfolds and retains in ER, or escapes from ER (2A) where they are recognized by golgi based ER Mannosidase 1 and translocated back to ER (2B) for ER Associated Degradation pathway(ERAD; 2C). Small molecules like SAHA could help in proper folding of misfolded AAT and can secrete it to serum (green arrow; potential therapy). 3. Misfolded AAT forms intermediates faster than WT, which is polymerogenic in nature. This accumulation cause liver cirrhosis. Small heat shock protein molecules are identified that could force intermediates to revert back to native misfolded state where they will be degraded by ERAD (green arrow, potential therapy). 4. Intermediates form toxic polymers and eventually ER becomes filled with toxic globules. This enhances autophagy that programs for self-destruction and in that way gets rid-off the toxic polymers. Enhancing autophagy by manipulating cellular signaling or by drugs like rapamycin could help in eliminating toxic globules (green arrow, potential therapy). Targeting $\mathrm{m}$ RNA by miRNA or anti-sense small oligonucleotide could also reverse the liver disease (green arrow, potential therapy). Figure provided by Richard Sifers.

TFEB is a promising candidate to clear the toxic polymers of A1AT Z protein from liver by enhancing autophagy. He studied the TFEB gene incorporated into Helper Dependent Adenoviral (HDAd) and the effect of its hepatocyte specific hepatocyte expression following intravenous injection in PiZ mouse model of A1AT liver disease. Use of this vector is advantageous over others due to the reason that they are non-integrating, do not contain viral coding sequences, have large cloning capacity, and result in long-term TFEB expression. After HDAd-TFEB injection, livers of PiZ mice showed decreased hepatic A1AT Z load. This decrease is associated with the increased expression of markers of autophagy. Immuno-label electron microscopy experiments showed that A1AT Z was targeted to the autophagolysosomes. A1AT mutant $\mathrm{Z}$ monomer and polymer molecules were significantly decreased in the livers of HD-Ad-TFEB injected PiZ mice. Furthermore, HDAd-TFEB injected PiZ mice had reduced liver inflammation, apoptosis, and fibrosis in livers. Further development as a therapeutic intervention for A1AT liver disease is proposed. Another therapeutic strategy of enhanced autophagy was proposed by Jeffrey Teckman, who discussed studies of norUDCA, a bile acid which when given in pharmacologic doses to the PiZ mouse model induces autophagy. Under high dose norUDCA, A1AT mutant Z globules disappear and fewer polymers are formed. Markers of liver injury, including apoptotic markers and fibrosis are also reduced. Future studies will compare the activity of norUDCA to Ursodeoxycholic Acid (UDCA), which is already approved for human use in other liver diseases, with the aim of medicinal development and possible human trial design.

\section{RNA technology and gene therapy}

Single gene diseases, such as A1AT, in which a single mutation accounts for the vast majority of disease; have been seen as attractive candidates for gene therapy and RNA therapeutics. However, useful extension of gene therapy to the 
clinic has not yet been fully realized. Past reports have shown that mutant $\mathrm{Z}$ protein in the liver of the PiZ model mouse can be reduced by ribozyme knock down of A1AT mutant Z mRNA and siRNA administration to reduce A1AT gene expression. Christian Mueller presented new studies in the transgenic PiZ mouse model using recombinant Adeno-Associated Virus (rAAV) vector designed with both the WT M A1AT to provide synthesis of normal, physiologic amounts of M A1AT to prevent lung disease and containing anti-A1AT mutant Z miRNA to prevent liver disease [36-38]. The strategy resulted in serum increased WT A1AT levels, which remained for at least a year. Decreased A1AT mutant $\mathrm{Z}$ accumulation in the liver, along with associated decreases in liver injury was also seen.

Another interesting approach to target liver injury by A1AT mutant Z is the use of Anti-Sense Oligonucleotides (ASO) to specifically degrade a target mRNA. Michael McCaleb [39] reported studies performed in industry, using ASO to reduce hepatic A1AT mutant Z protein synthesis and thereby reduce liver injury. The ASO binds the A1AT mRNA specifically, and targets it for destruction through RNAseH mediated degradation. The studies in the PiZ mouse model show rapid, dose-dependent, and powerful inhibition of A1AT mutant Z protein synthesis, and reductions in the hepatic mutant $\mathrm{Z}$ protein burden. This was associated with reduced markers of liver injury and fibrosis. Future developments for human trials is feasible, but are complicated by questions of proper trial design in a chronic liver disease such as this, in which there are large numbers of asymptomatic individuals.

\section{Cell replacement and stem cell therapies}

Liver cell replacement therapies have also been widely proposed for metabolic liver diseases, such as A1AT. Bruno Christ [40] reported novel approaches in which stem cell based hepatocyte differentiation could be used as a source of cells for hepatocellular transplantation in liver therapy. He discussed transplantation of murine Mesenchymal Stem Cells (MSC) in the NASH mouse model. Transplantation of MSC in these mice resulted in successful proliferation and differentiation of stem cells as hepatocytes post-transplantation. The levels of hepatic lipid are decreased markedly five weeks after transplantation. Christ also discussed the therapeutic potential of adipose tissue derived human MSC in NASH mouse liver. A marked decrease in inflammatory markers followed by an attenuation of hepatic fibrosis has been observed within a week of transplantation. Studies have now begun to examine the utility of this strategy in the PiZ mouse model of A1AT deficiency. Stuart Forbes also presented commentary and data on liver cell proliferation in disease pathophysiology and therapy. During proliferation in the ZZ liver, proliferation is part of the response to injury, but may also be a contributing factor to the development of fibrosis. Modulation of this process is being studies intensely in many liver diseases.

Studies using Human induced Pluripotent Stem Cells (HiPSCs) by Andrew Wilson were presented showing that iPSCs from monocytes of A1AT deficient individuals could be used to reprogram and correct the lung disease phenotype. iPSCs obtained from individuals who have pulmonary disease due to A1AT are grown in media for twenty five days. Preliminary evidence in mouse model studies showed that both iPSCs in WT and PiZ results in increased production of intracellular corrected A1AT (66\% in WT and $88 \%$ in PiZ). Studies are now being designed to translate this attractive strategy to iPSCs based human trials.

\section{Liver fibrosis as a therapeutic target}

In the final session of the meeting, David Brenner summarized therapies for liver injury focused on Hepatic Stellate Cells (HSC) examined in various experimental systems. HSCs respond to injury in the five stages of activation, perpetuation, regression, inflammation, and inactivation of HSCs $[41,42]$. HSCs are induced by Peroxysome Proliferator Activated Receptor (PPAR $\delta$ ) a class of nuclear receptor which is induced in liver under stress and have pleiotropic effects in response to injury. Activated HSCs can result in the trans-differentiation of myofibroblasts as well as deposition of extracellular-matrix proteins in order to initiate cellular apoptosis as a response to fibrotic damage. Although beneficial in nature, these changes along with stress condition in damaged liver are extremely vulnerable to subtle differences in gene expressions controlled by PPAR class of proteins. Failure of tight regulation of these events leads to cell death, fibrosis, and HCC. Given the important roles played by PPAR $\delta$, this could be targeted to inhibit abnormal induction of the HSCs. In this context, KD3010, a potent agonist of PPAR $\delta$, and other agents have been able to protect hepatocytes from cell death (in the model liver injury induced by $\mathrm{CCl}_{4}$ ) in cell-culture. Studies are underway to determine if these effects can be extended to models of metabolic disease.

The second stage of liver injury is the proliferation of HSCs, which is induced by several growth-factors like PDGF and VDGF tyrosine kinases. These growth factors could also be targeted to lessen the liver injury. Additionally, renin-angiotensin system induces reactive oxygen species in HSCs resulting in induction of nicotinamide adenine dinucleotide phosphate oxidase (NOX, NOX2, NOX4). These molecules are also known to induce the hepatic failure. GKT137831is a potent antagonist of NOX1-4 is capable of reducing liver fibrosis [43].

The third and fourth stage is the induction and perpetuation of fibrosis of liver in which matrix enzyme Lysil-oxidase-Like 2 (LOXL-2) plays a crucial role. Monoclonal antibody directed against LOXL-2 has an inhibitory effect and thus reduces hepatic fibrosis in some model systems [43].The fifth stage of the disease is regression of hepatic injury. This is further divided in two stages, apoptosis and inactivation. HSCs generate TIMP1 protein that induces endogenous collagen production and apoptosis. Monoclonal antibody against TIMP1 inhibits its activity and partially reverses liver cirrhosis in $\mathrm{CCl}_{4}$ induced mouse model of liver injury. Studies are ongoing to extend these discoveries to human trials and to metabolic diseases such as A1AT in which the cell death and resultant proliferative stimulus is low but constant. 


\section{Conclusions}

Homozygous ZZ A1AT deficiency is a common genetic liver disease which can affect adults and children. The clinical manifestations are highly variable, with many patients remaining healthy or exhibiting only mild biochemical abnormalities until late in life. Accumulation of the A1AT mutant Z protein within hepatocytes activates an intracellular injury cascade of apoptotic liver cell death and compensatory hepatocellular proliferation leading to end organ injury. Genetic and environmental disease modifiers are thought to be important, but are still poorly understood. There is no specific treatment for A1AT associated liver disease, but there are treatment options involving supportive measures and liver transplant. New technologies aimed at stimulating proteolysis pathways, small molecule chaperones, gene therapy, RNA technologies, cell transplantation, or anti-fibrotic therapies may hold promise for the treatment of this disease. Future research is likely to lead to studies of these new approaches, although the high degree of clinical variability will pose a challenge to the design of clinical trials.

\section{Acknowledgements}

We thank John W. Walsh, president and CEO of Alpha-1 Foundation and Dr. Adam Wanner, Scientific Director of Alpha-1 Foundation for organizing the meeting. We would also like to extend our sincere gratitude to Dr. Marc Miravitlles, Dr. Rob Stockley, and Dr. Jeff Teckman for serving as scientific committee. This conference was supported by the Alpha 1 Foundation and Grifols.

\section{References}

1. American Thoracic Society; European Respiratory Society. American Thoracic Society/European Respiratory Society Statement: Standards for the Diagnosis and Management of Individuals with Alpha-1 Antitrypsin Deficiency. Am J Respir Crit Care Med. 2003;168(7): 818900.

2. Eriksson S. Alpha-1-antitrypsin deficiency: natural course and therapeutic strategies. In: Boyer JL, editor. Liver Cirrhosis and its Development; Volume 115 of Falk Symposium. london: Springer Science \& Business Media. 2001; 307-315.

3. Nelson DR, Teckman J, Di Bisceglie AM, Brenner DA. Diagnosis and Management of Patients With $\alpha 1$-Antitrypsin (A1AT) Deficiency. Clin Gastroenterol Hepatol. 2012; 10(6): 575-80. doi: 10.1016/j. cgh.2011.12.028.

4. Rudnick DA, Liao Y, An JK, Muglia LJ, Perlmutter DH, Teckman JH Analyses of hepatocellular proliferation in a mouse model of alpha-1antitrypsin deficiency. Hepatology. 2004; 39(4): 1048-1055.

5. Lindblad D, Blomenkamp K, Teckman J. Alpha-1-antitrypsin mutant Z protein content in individual hepatocytes correlates with cell death in a mouse model. Hepatology. 2007; 46(4): 1228-1235.

6. Eriksson S. Discovery of alpha 1-antitrypsin deficiency. Lung. 1990;168 Suppl: 523-529.

7. Sveger T. Liver disease in alpha1-antitrypsin deficiency detected by screening of 200,000 infants. N Engl J Med. 1976; 294(24): 1316-1321.

8. Bernspang E, Carlson J, Piitulainen E. The liver in 30-year-old individuals with alpha(1)-antitrypsin deficiency. Scand J Gastroenterol.

\section{9;44(11): 1349-1355. doi: 10.3109/00365520903296669.}

9. Sharp HL. The current status of alpha-1-antityrpsin, a protease inhibitor, in gastrointestinal disease. Gastroenterology. 1976; 70 (4): 611-621.

10. Wu Y, Whitman I, Molmenti E, Moore K, Hippenmeyer P, Perlmutter DH. A lag in intracellular degradation of mutant alpha 1-antitrypsin correlates with the liver disease phenotype in homozygous PiZZ alpha 1-antitrypsin deficiency. Proc Natl Acad Sci USA. 1994; 91(19): $9014-$ 9018.

11. Sifers RN. Cell biology. Protein degradation unlocked. Science. 2003;299(5611): 1330-1331.

12.Sifers RN. Medicine. Clearing conformational disease. Science. 2010;329(5988): 154-155. doi: 10.1126/science.1192681.

13. Lomas DA, Evans DL, Finch JT, Carrell RW. The mechanism of Z alpha 1-antitrypsin accumulation in the liver. Nature. 1992; 357(6379): 605-607.

14. Teckman JH, Perlmutter DH. Retention of mutant alpha(1)-antitrypsin $\mathrm{Z}$ in endoplasmic reticulum is associated with an autophagic response. Am J Physiol Gastrointest Liver Physiol. 2000; 279(5): 961-974.

15. James EL, Bottomley SP. The mechanism of alpha 1-antitrypsin polymerization probed by fluorescence spectroscopy. Arch Biochem Biophys. 1998;356(2): 296-300.

16. Lomas DA, Mahadeva R. Alpha1-antitrypsin polymerization and the serpinopathies: pathobiology and prospects for therapy. J Clin Invest. 2002; 110(11): 1585-1590.

17. Qu D, Teckman JH, Omura S, Perlmutter DH. Degradation of a mutant secretory protein, alpha1-antitrypsin Z, in the endoplasmic reticulum requires proteasome activity. J Biol Chem. 1996; 271(37): 2279122795.

18. Qu D, Teckman JH, Perlmutter DH. Review: alpha 1-antitrypsin deficiency associated liver disease. J Gastroenterol Hepatol. 1997; 12(5): 404-416

19. Cabral CM, Choudhury P, Liu Y, Sifers RN. Processing by endoplasmic reticulum mannosidases partitions a secretion-impaired glycoprotein into distinct disposal pathways. J Biol Chem. 2000; 275(32): 2501525022 .

20. Teckman JH, Burrows J, Hidvegi T, Schmidt B, Hale PD, Perlmutter DH. The proteasome participates in degradation of mutant alpha 1-antitrypsin $\mathrm{Z}$ in the endoplasmic reticulum of hepatoma-derived hepatocytes. J Biol Chem. 2001; 276(48): 44865-44872.

21. Dafforn TR, Mahadeva R, Elliott PR, Sivasothy P, Lomas DA. A kinetic mechanism for the polymerization of alpha1-antitrypsin. J Biol Chem. 1999; 274(14): 9548-9555.

22. Bottomley SP. The structural diversity in alpha1-antitrypsin misfolding. EMBO Rep. 2011;12(10): 983-984.

23. Knaupp AS, Bottomley SP. Structural change in beta-sheet A of Z alpha(1)-antitrypsin is responsible for accelerated polymerization and disease. J Mol Biol. 2011; 413(4): 888-898.

24. Wu Y, Swulius MT, Moremen KW, Sifers RN. Elucidation of the molecular logic by which misfolded alpha 1-antitrypsin is preferentially selected for degradation. Proc Natl Acad Sci USA. 2003; 100(14): 8229-8234.

25. Teckman JH, Gilmore R, Perlmutter DH. Role of ubiquitin in proteasomal degradation of mutant alpha(1)-antitrypsin $\mathrm{Z}$ in the endoplasmic reticulum. Am J Physiol Gastrointest Liver Physiol. 2000; 
278(1): 39-48.

26. Iannotti MJ, Figard L, Sokac AM, Sifers RN. A Golgi-localized mannosidase (MAN1B1) plays a non-enzymatic gatekeeper role in protein biosynthetic quality control. J Biol Chem. 2014; 289(17): 11844-11858. doi: 10.1074/jbc.M114.552091.

27.Pan S, Cheng X, Sifers RN. Golgi-situated endoplasmic reticulum alpha-1, 2-mannosidase contributes to the retrieval of ERAD substrates through a direct interaction with gamma-COP. Mol Biol Cell. 2013; 24(8): 1111-1121. doi: 10.1091/mbc.E12-12-0886.

28. Teckman JH, Perlmutter DH. The endoplasmic reticulum degradation pathway for mutant secretory proteins alpha1-antitrypsin $\mathrm{Z}$ and $\mathrm{S}$ is distinct from that for an unassembled membrane protein. J Biol Chem. 1996; 271(22): 13215-13220.

29. Cabral CM, Liu Y, Moremen KW, Sifers RN. Organizational diversity among distinct glycoprotein endoplasmic reticulum-associated degradation programs. Mol Biol Cell. 2002; 13(8): 2639-2650.

30. Pan S, Huang L, McPherson J, Muzny D, Rouhani F, Brantly M, et al. Single nucleotide polymorphism-mediated translational suppression of endoplasmic reticulum mannosidase I modifies the onset of endstage liver disease in alpha1-antitrypsin deficiency. Hepatology. 2009; 50(1): 275-281. doi: 10.1002/hep.22974.

31. Powers ET, Balch WE. Protein folding: Protection from the outside Nature. 2011; 471(7336): 42-43. doi: 10.1038/471042a.

32. Bouchecareilh M, Hutt DM, Szajner P, Flotte TR, Balch WE. Histone deacetylase inhibitor (HDACi) suberoylanilide hydroxamic acid (SAHA)-mediated correction of alpha1-antitrypsin deficiency. J Biol Chem. 2012; 287(45): 38265-38278. doi: 10.1074/jbc.M112.404707.

33. Kaushal S, Annamali M, Blomenkamp K, Rudnick D, Halloran D, Brunt EM, et al. Rapamycin reduces intrahepatic alpha-1-antitrypsin mutant $\mathrm{Z}$ protein polymers and liver injury in a mouse model. Exp Biol Med (Maywood). 2010; 235(6): 700-709. doi: 10.1258/ebm.2010.009297.

34. Hidvegi T, Ewing M, Hale P, Dippold C, Beckett C, Kemp C, et al. An autophagy-enhancing drug promotes degradation of mutant alpha1antitrypsin Z and reduces hepatic fibrosis. Science. 2010; 329(5988): 229-232. doi: 10.1126/science.1190354
35. Pastore N, Ballabio A, Brunetti-Pierri N. Autophagy master regulator TFEB induces clearance of toxic SERPINA1/alpha-1-antitrypsin polymers. Autophagy. 2013; 9(7): 1094-1096. doi: 10.4161/ auto. 24469 .

36. Mueller C, Flotte TR. Gene-based therapy for alpha-1 antitrypsin deficiency. COPD. 2013; 10 Suppl 1: 44-49. doi: 10.3109/15412555.2013.764978.

37. Mueller C, Chulay JD, Trapnell BC, Humphries M, Carey B, Sandhaus RA, et al. Human Treg responses allow sustained recombinant adenoassociated virus-mediated transgene expression. J Clin Invest. 2013; 123(12): 5310-5318. doi: 10.1172/JCI70314.

38. Mueller C, Tang Q, Gruntman A, Blomenkamp K, Teckman J, Song L, et al. Sustained miRNA-mediated Knockdown of Mutant AAT With Simultaneous Augmentation of Wild-type AAT Has Minimal Effect on Global Liver miRNA Profiles. Mol Ther. 2012; 20(3): 590-600. doi: 10.1038/mt.2011.292.

39. Guo S, Booten SL, Aghajan M, Hung G, Zhao C, Blomenkamp K, et al. Antisense oligonucleotide treatment ameliorates alpha-1 antitrypsinrelated liver disease in mice. J Clin Invest. 2014; 124(1): 251-261. doi: 10.1172/JCI67968.

40. Pelz S, Stock P, Bruckner S, Christ B. A methionine-choline-deficient diet elicits NASH in the immunodeficient mouse featuring a model for hepatic cell transplantation. Exp Cell Res. 2012; 318(3): 276-287. doi: 10.1016/j.yexcr.2011.11.005

41. Iwaisako K, Haimerl M, Paik YH, Taura K, Kodama Y, Sirlin C, et al. Protection from liver fibrosis by a peroxisome proliferator-activated receptor delta agonist. Proc Natl Acad Sci U S A. 2012; 109(21): E13691376. doi: 10.1073/pnas.1202464109.

42. Iwaisako K, Brenner DA, Kisseleva T. What's new in liver fibrosis? The origin of myofibroblasts in liver fibrosis. J Gastroenterol Hepatol. 2012; 27 Suppl 2: 65-68. doi: 10.1111/j.1440-1746.2011.07002.x.

43. Aoyama T, Paik YH, Watanabe S, Laleu B, Gaggini F, Fioraso-Cartier $\mathrm{L}$, et al. Nicotinamide adenine dinucleotide phosphate oxidase in experimental liver fibrosis: GKT137831 as a novel potential therapeutic agent. Hepatology. 2012; 56(6): 2316-2327. doi: 10.1002 / hep. 25938 